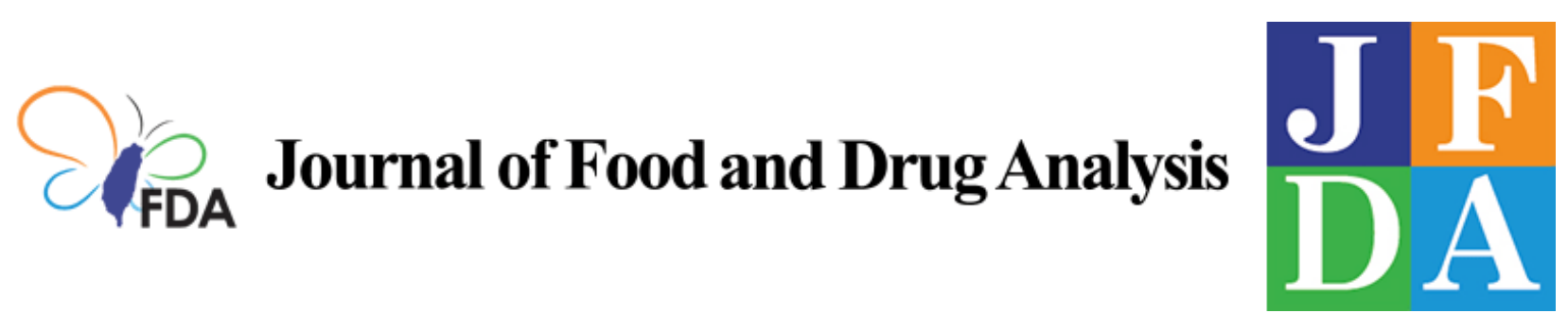

Volume 28 | Issue 2

Article 4

2020

\title{
Screening for malachite green contamination on live fish skin with chewing gum based viscoelastic SERS sensor
}

Follow this and additional works at: https://www.jfda-online.com/journal

Part of the Food Science Commons, Medicinal Chemistry and Pharmaceutics Commons, Pharmacology Commons, and the Toxicology Commons

(c) (i) (9)

This work is licensed under a Creative Commons Attribution-Noncommercial-No Derivative Works 4.0 License.

\section{Recommended Citation}

Fan, Wan-Li; Yang, Shi-Wei; Wang, Dong-Mei; Gong, Zheng-Jun; and Fan, Mei-Kun (2020) "Screening for malachite green contamination on live fish skin with chewing gum based viscoelastic SERS sensor," Journal of Food and Drug Analysis: Vol. 28 : Iss. 2 , Article 4.

Available at: https://doi.org/10.38212/2224-6614.1057

This Original Article is brought to you for free and open access by Journal of Food and Drug Analysis. It has been accepted for inclusion in Journal of Food and Drug Analysis by an authorized editor of Journal of Food and Drug Analysis. 


\title{
Screening for malachite green contamination on live fish skin with chewing gum based viscoelastic SERS sensor
}

\author{
Wan-Li Fan ${ }^{a, 1}$, Shi-Wei Yang ${ }^{b, 1}$, Dong-Mei Wang ${ }^{a, *}$, \\ Zheng-Jun Gong ${ }^{a}$, Mei-Kun Fan ${ }^{\mathrm{a}, \mathrm{b}, * *}$ \\ a Faculty of Geosciences and Environmental Engineering, Southwest Jiaotong University, Chengdu, Sichuan 610031, China
b School of Civil Engineering, Southwest Jiaotong University, Chengdu, Sichuan 610031, China
}

Abstract

Malachite green (MG), a prohibited but still found antimicrobial in aquafarm and during live fish shipping, is a hot target in food safety screening. Herein, a novel chewing gum based flexible SERS (G-SERS) sensor was proposed for rapid sampling and detection of MG on live fish skin. The whole analysis takes $<5$ min, while the limit of detection for MG is $0.73 \mathrm{pg}$. Different from other reports, MG contaminated live fish was monitored daily with the G-SERS sensor, during which the fish was firstly raised in $0.5 \mathrm{ppm}$ MG solution for one day, followed by freshwater for a week. It was found that the SERS signal of residue MG on fish skin could still be seen even on the sixth day, roughly the sale cycle of live fish in a marketplace. Furthermore, the method was also applied for MG screening on the skin of fish purchased from a supermarket and a local street marketplace. MG was found on some fishes from the latter but not from the former, which was cross-validated by LC-MS, suggesting MG risks still exist in smaller marketplaces. This work demonstrated the feasibility of using the flexible SERS sensor for onsite food safety screening.

Keywords: Food safety monitoring, Malachite green, Live fish, SERS, Gum

\section{Introduction}

$I^{2}$ $\mathrm{n}$ recent years, formulated feeds (containing antibiotics, antifungal drugs, and other pharmaceuticals), pesticides and disinfectants have been widely used in aquaculture [1]. It may raise some serious concerns on the safety of seafood [2]. For example, antibacterials have been frequently detected in fish products [3,4], which is known to cause environmental and health issues (e.g., antibiotic resistance for bacteria [5]). Malachite green (MG), a good antibacterial but with potential carcinogenicity, mutagenicity, and teratogenicity, is prohibited in many countries [6,7]. In fact, it is one of the regulated inspection items for aquatic products globally because it is still used illegally owing to its low cost and high effectiveness, particularly in developing countries [8]. What's worse, the culture of pursuing live fish in countries like China has put a lot of stress on the supply chain, which could lead to the abuse of MG to improve the survival rate during live fish shipping, and hence presents the MG exposure risk right on the dinner table [9]. Therefore, onsite MG screening on live fish during shipping or in the market is very important to ensure food safety.

The primary analytical methods to detect MG include high-performance liquid chromatography (HPLC) [10] and liquid chromatography-tandem mass spectrometry (LC-MS) [6], which have high

Received 11 December 2019; accepted 30 January 2020.

Available online 27 June 2020

* Corresponding author at: Faculty of Geosciences and Environmental Engineering, Southwest Jiaotong University, Chengdu, Sichuan 610031, China.

** Corresponding author at: Faculty of Geosciences and Environmental Engineering, Southwest Jiaotong University, Chengdu, Sichuan 610031, China.

E-mail addresses: dongmeiwang@home.swjtu.edu.cn (D.-M. Wang), meikunfan@gmail.com (M.-K. Fan).

1 These authors contributed equally to this work. 
detection accuracy. Nevertheless, at least two issues are related to these methods. For one, the time frame from the aquafarm to the dining table (shipping plus sales cycle) is usually short (roughly less than a week); for another, the culture of pursuing live fish demands local purchasing from the decentralized street marketplaces. The lengthy pretreatment procedure and bulky instruments could seriously compromise the safeguarding role of these methods. That is, when the (positive) test results came back, the fish had probably already gone on the dining table. Therefore, fast on-site screening of MG is very necessary, especially in countries like China, where there are many decentralized street marketplaces and has a strong traditional culture for live fish.

Surface-enhanced Raman scattering (SERS) is considered to be one of the most important molecular spectroscopic techniques and suitable for onsite analysis [11,12]. In fact, there are many reports about the detection of MG on seafood based on SERS [1,8,13-24]. Nevertheless, the effectiveness of the SERS methods remains to be verified. For example, MG in fish tissues could be detected with SERS $[18,19,21,24]$, but it is not suitable for onsite screening. There are also groups who devoted to the sensing of MG on the live fish by applying MG solution directly onto the body of an aquatic product $[8,14-16,20]$, or soaking the aquatic products into diluted MG solution [1,17,22,23]. However, neither of the exposing scenarios is the general practice of illegal MG use, which usually involves raising the fishes in ppm level of MG solution during shipping (up to $36 \mathrm{~h}$ ), followed by raising them in freshwater before the sale [7,9]. MG residue (if any) will undergo degradation and dissolving with time (during the time in the market). The effectiveness of SERS for MG sensing in this real-life scenario has not been explored, though it is vital for MG contamination risk evaluation.

Chewing gum of which the main ingredient is gum Arabic can undergo a large deformation [25]. Thus, it has been used as a stretchable and foldable sensor for monitoring muscle and joint motions [26]. On the other hand, gum has high viscoelasticity which means good adhesion capacity to remove any residual contaminants (analytes) from a surface. Herein, chewing gum was used for the fabrication of a flexible, adhesive and sensitive hydrogel SERS sensor to detect MG on live fish. Citrate-reduced Ag nanoparticles (Ag NPs) were prepared and modified with various halogen anions, including $\mathrm{I}^{-}, \mathrm{Br}^{-}$and $\mathrm{Cl}^{-}$. It was found that $\mathrm{I}^{-}$could give the highest enhancement for MG. Thus, the Ag NPs-KI mixture was then mixed with the gum to form the SERS sensor. The preparation procedure was shown in Scheme 1. The sensitivity and recovery efficiency for analyte MG, viscoelasticity, shelf-life, and reproducibility of the prepared sensor were explored. Later, the G-SERS sensor was applied for the detection of MG on the fish body. In the process, the crucian carp was firstly raised in MG spiked water for one day, and then in freshwater for a week, the G-SERS sensor was used to monitor the residual MG on the fish body each day. Finally, the proposed method was applied to the screening of MG residues on the crucian carp purchased from a supermarket and a local street marketplace before verifying with results obtained from the standard LC-MS method.

\section{Material and methods}

\subsection{Reagents and materials}

Silver nitrate $(99 \%)$, sodium citrate $(99 \%)$, Nile blue A (NBA), rhodamine 6G (R6G) and MG were supplied by Sigma-Aldrich (Shanghai, China). Potassium iodide, potassium bromide, potassium chloride, and ethanol were purchased from Shanghai Titan Scientific Co., Ltd, China. Doublemint ${ }^{\circledR}$ gum was obtained from a local supermarket. 18.2 $\mathrm{M} \Omega \mathrm{cm}$ water (Nanopure, Thermo) was used throughout the experiment.

\subsection{Instruments}

SERS measurements were performed on a customized Raman microscope equipped with Acton SP-2500i spectrograph (Princeton Instrument, US) and Pixis-100BR CCD (Princeton Instrument, US) in the whole experiment. $\mathrm{A} \mathrm{He}-\mathrm{Ne}$ laser $(14 \mathrm{~mW})$ was used in the experiments. Laser power $12 \times \mathrm{mW}, 50 \times$ objective, and $5 \mathrm{~s}$ integration time were used in the experiments. At least 10 spots on the same G-SERS sensor were examined. The Ag NPs was centrifuged in a centrifuge (Microfuge ${ }^{\circledR}$ $22 \mathrm{R}$, Beckman coulter, US) at 10,000 rpm for $10 \mathrm{~min}$ at $20^{\circ} \mathrm{C}$. The Ag NPs were characterized by a Field Emission Transmission Electron Microscope (TEM, JEM-2100 F, Japan), and the gum and G-SERS sensor by a scanning electron microscope (SEM, FEI Inspect F), respectively. The sampling recovery efficiency was determined by a UV-Vis spectrophotometer (UV-Vis, UV-4802H, China) at the wavelength of $614 \mathrm{~nm}$. Liquid chromatographymass spectrometry (LC-MS) measurements were performed on Shimadzu LCMS-8045 (USA). 


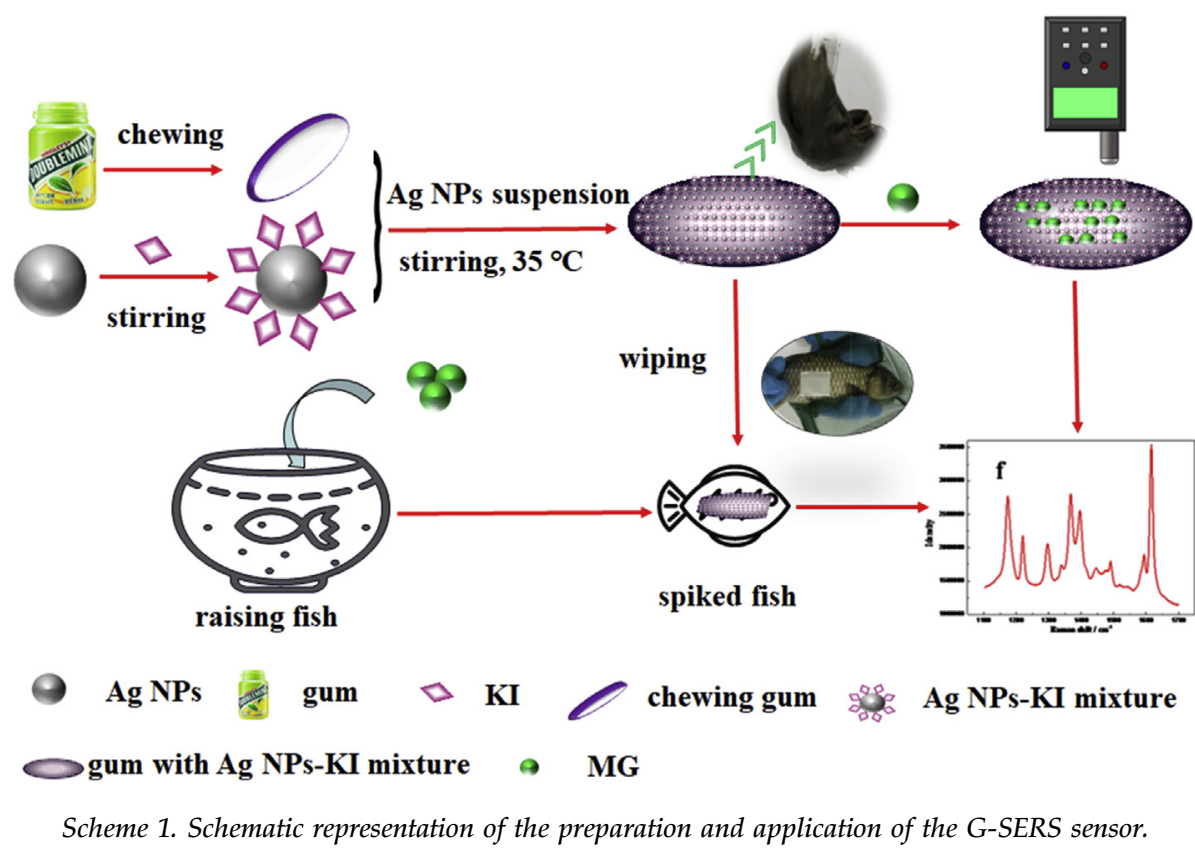

\subsection{Recommended procedures}

\subsubsection{Preparation of $A g N P s$}

Silver nitrate was used to prepare Ag NPs [27]. Briefly, $100 \mathrm{~mL}$ of $\mathrm{AgNO}_{3}(5 \mathrm{mM})$ solution was heated to boiling with rapid stirring. $2 \mathrm{~mL}$ of $10 \%$ sodium citrate aqueous solution was added quickly, and the mixture was boiled for another $1 \mathrm{~h}$. Finally, the solution was cooled to room temperature, denoted as $5 \mathrm{mM} \mathrm{Ag} \mathrm{NPs.}$

\subsubsection{Preparation of G-SERS sensor}

Firstly, gum was chewed for about $30 \mathrm{~min}$ [26]. It was then washed with ethanol and ultrapure water for about $5 \mathrm{~min}$, respectively. Then, it was heated to $35^{\circ} \mathrm{C}$ for $1 \mathrm{~h}$ to evaporate the excess amount of water from the gum. Meanwhile, a certain volume of $5 \mathrm{mM}$ Ag NPs (the optimization was shown in "3.2. Optimization of the SERS Sensor") was centrifuged and the supernatant was removed. The remaining mixture in the centrifuge tube was about $100 \mu \mathrm{L}$. Then, $10 \mu \mathrm{L}$ of $100 \mathrm{mM}$ halogen ion solution was added to the $100 \mu \mathrm{L}$ concentrated Ag NPs and the mixture was vortexed for $1 \mathrm{~min}$. Immediately, the Ag NPs-halogen ion mixture was added to $0.3 \mathrm{~g}$ chewing gum, which was heated to $35^{\circ} \mathrm{C}$ for another $30 \mathrm{~min}$. The chewing gum was repeatedly folded and stretched during this period with a glass rod. The fabrication process involves no toxic chemicals.

\subsubsection{SERS analysis}

In this work, there were two protocols: (1) For optimization and characterization of the G-SERS sensor: $5 \mu \mathrm{L}$ MG aqueous solution was dropped on about $0.1 \mathrm{~g}$ G-SERS sensor, which was subjected to SERS analysis after the solvent evaporated. (2) For the daily monitoring of the sensor: ten crucian carp (Carassius carassius) from the local supermarket were randomly divided into two groups: the experimental group and the control group. The control group was raised in a $75 \mathrm{~L}$ aquarium with tap water for a week. The experiment group was first exposed to 0.5 ppm MG aqueous solution [7] in another $75 \mathrm{~L}$ tank for one day and then raised in the tap water for a week (Fig. S1C) as well. In the process, water was changed daily and $\mathrm{O}_{2}$ was supplied for both groups. The G-SERS sensor was used to stamp on the fish body (Fig. S1B) and then was analyzed on the Raman microscope daily for 6 days. Finally, MG on the crucian carp from 4 stalls in a street marketplace and a supermarket was monitored on the same day when purchased by this method, which was crossvalidated by LC-MS approach [28] (supporting information).

\subsubsection{Sample recovery efficiency (SRE) analysis}

The SRE of the G-SERS sensor for MG was determined by a UV-Vis spectrophotometer [29]. Briefly, $20 \mu \mathrm{L}$ of MG aqueous solution was dropped on a frozen fish. After $10 \mathrm{~min}$, the G-SERS sensor was used to stamp on the fish body to recover the MG. Finally, the sensor was placed in a beaker filled with $10 \mathrm{~mL}$ of deionized water and sonicated for $20 \mathrm{~min}$ to extract the MG. The experimental conditions for the UV-Vis analysis can be found in Fig. S2. 


\section{Results and discussion}

\subsection{Preparation and characterization of the G- SERS sensor}

The fabrication of the G-SERS sensor is very straightforward: citrate-reduced Ag NPs (Fig. 1A) were prepared and then modified with KI. Finally, the Ag NPs-KI mixture was doped in the gum to form the SERS sensor (Schemes 1 and S1A). The gum and G-SERS sensor were characterized by SEM, which were shown in Fig. 1B and C, respectively. It was clear that scattering NPs aggregates can be found in Fig. 1C. Besides, the color of the latter (the inset in Fig. 1C) became dark compared with the former (the inset in Fig. 1B), showing that gum was successfully doped with Ag NPs (Fig. 1C).

\subsection{Optimization and characterization of the SERS sensor}

Reports have shown that Ag NPs modified with iodide ion had a significant effect on the SERS signals of some analytes [30]. Herein, the same strategy was used to fabricate the G-SERS sensor through Ag NPs modified with KI. The SERS spectra in Fig. 2A showed the effect of KI on the signal of MG. It was found that the G-SERS sensor for the analysis of MG had an evident signal enhancement in the presence of gum, Ag NPs and KI. And the G-SERS sensor was found to have negligible interference with MG due to its clean Raman background. For MG, the major Raman peaks appeared at 1175,1369 and $1618 \mathrm{~cm}^{-1}$ were ascribed to ring $\mathrm{C}-\mathrm{H}$ in-plane bending $\left(1175 \mathrm{~cm}^{-1}\right), \mathrm{N}$-phenyl stretching (1369 $\left.\mathrm{cm}^{-1}\right)$ and ring $\mathrm{C}-\mathrm{C}$ stretching $\left(1618 \mathrm{~cm}^{-1}\right)$, respectively [31,32]. We also explored the effect of remaining halogen ions on SERS performance. The SERS spectra of MG on the G-SERS sensors in the presence of $\mathrm{KCl}, \mathrm{KBr}$, and $\mathrm{KI}$ were shown in Fig. 2B, respectively. It was found that GSERS sensor fabricated with KI modified Ag NPs had the highest intensity. It's believed that $\mathrm{I}^{-}$may change the shape of the silver surface in addition to inducing the aggregation to achieve a better signal [33]. Thus, we decide to use KI modified Ag NPs in the preparation of G-SERS sensor in the following experiment.

We then tried to optimize the concentration of $\mathrm{I}^{-}$in the G-SERS sensor. To address this, $2-80 \mu \mathrm{L}$ of $100 \mathrm{mM} \mathrm{KI}$ was mixed with $10 \mathrm{~mL} \mathrm{Ag} \mathrm{NPs,} \mathrm{and} \mathrm{the}$ mixture was mixed with $0.3 \mathrm{~g}$ gum for preparing the G-SERS sensor, respectively. The results were shown in Fig. 2C. Obviously, the signal of MG increased firstly and then decreased with the augment of the amount of KI. It was found that the G-SERS sensor mixed with $10 \mu \mathrm{L}$ of $100 \mathrm{mM}$ KI had the best SERS signal (at $1619 \mathrm{~cm}^{-1}$ ). This is probably caused by Ag NPs over aggregation induced by excessive KI [30], which is known to be able to lower the SERS performance of given SERS sensor. Thus, $10 \mu \mathrm{L}$ of $100 \mathrm{mM} \mathrm{KI}$ was used in the following experiments.

After optimizing the amount of $\mathrm{I}^{-}$, the effect of $\mathrm{Ag}$ NPs concentration was then explored to get the optimal G-SERS sensor. In Fig. 2D, the SERS performance for MG at $1618 \mathrm{~cm}^{-1}$ was obtained with 0.3 g gum containing 3-25 mL of Ag NPs which was concentrated and modified with $10 \mu \mathrm{L}$ of $100 \mathrm{mM} \mathrm{KI}$, respectively. Apparently, the signal increased first and then gradually decline with the increase of $\mathrm{Ag}$ NPs. The best signal was achieved at the amount of $10 \mathrm{~mL} \mathrm{Ag} \mathrm{NPs} \mathrm{(before} \mathrm{centrifuge).} \mathrm{To} \mathrm{be} \mathrm{specific,}$ lower concentration of Ag NPs can not produce sufficient SERS hot spots (Fig. S3A), resulting weaker SERS signal [34]. The increase of the SERS signal before $10 \mathrm{~mL}$ was explained by increased $\mathrm{Ag}$ NPs aggregates with the increased amount of $\mathrm{Ag}$ NPs [13]. However, at volume larger than $10 \mathrm{~mL}$, over aggregation of Ag NPs (Fig. S3B) could happen, which is known to be inefficient in SERS peroformance [27].

In conclusion, the optimal procedure to fabricate the G-SERS sensor was as follows: $10 \mathrm{~mL} \mathrm{Ag} \mathrm{NPs}$ was concentrated and mixed with $10 \mu \mathrm{L}$ of $100 \mathrm{mM}$ $\mathrm{KI}$, and the mixture was then added into $0.3 \mathrm{~g}$ gum.

\subsection{Reproducibility and stability of G-SERS sensor}

The optimal G-SERS sensor was compressed with a glass slide to form a flat surface for testing the reproducibility of the gum sensor. Briefly, $5 \mu \mathrm{L}$ of $10 \mu \mathrm{M}$ aqueous solution of MG was added onto the sensor. This experiment was repeated with 10 gum sensors, and ten SERS spectra were recorded from each gum. Fig. 3A showed the results of these experiments. The relative standard deviation (RSD\%) was found to be $15 \%$ between different sensors and 9-25\% within a single G-SERS sensor, which was comparable to other reports [27]. In order to test the shelf life of the G-SERS sensor, $3 \mathrm{~g}$ of the G-SERS sensor was prepared and sealed in fridge at $4{ }^{\circ} \mathrm{C}$. And $0.1 \mathrm{~g}$ of the G-SERS sensor was cut and explored for its SERS performance each day for a week, respectively. Fig. 3B showed the variation of SERS intensity of MG at the peak of $1618 \mathrm{~cm}^{-1}$. The RSD of signal intensities was found to be less than $11 \%$ for 8 days. Meanwhile, there was no significant difference in SERS performance of the G-SERS sensor in 8 days based upon the $t$-test (Table S1), confirming good 


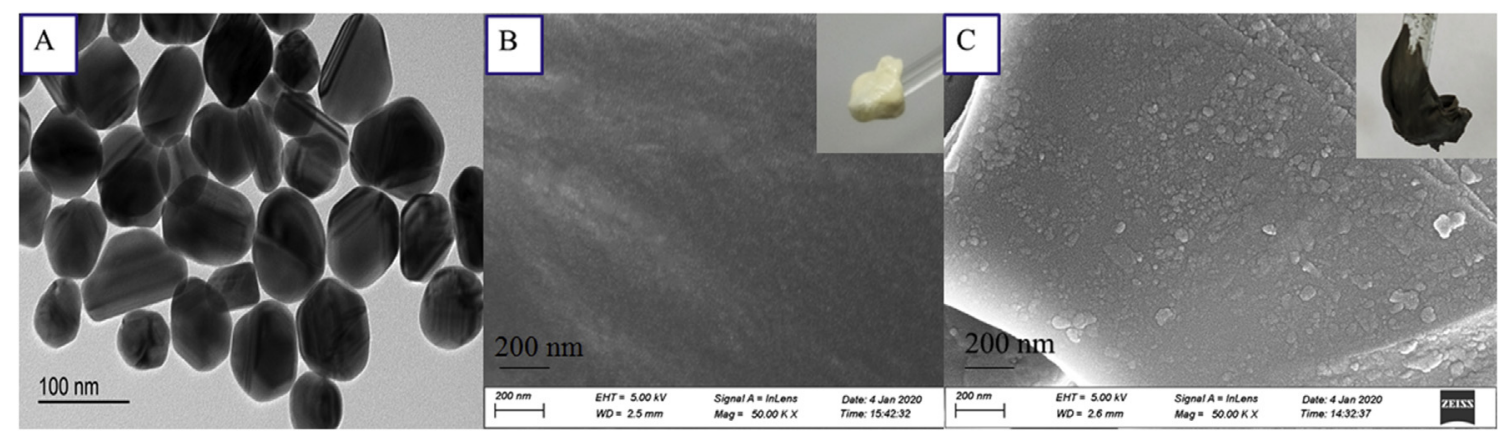

Fig. 1. TEM of Ag NPS (A), SEM images of gum (B) and G-SERS sensor (C). The insets in B and C are the photos of the gum and G-SERS sensor.

stability of G-SERS sensor. Hence, the G-SERS sensor has good reproducibility and stability.

\subsection{LOD of the G-SERS sensor}

To determine the SERS sensitivity, $5 \mu \mathrm{L}$ of various concentrations of MG aqueous solution were added onto the G-SERS sensor. The results were shown in Fig. 3C. It is clear that the characteristic peaks of MG at $1175 \mathrm{~cm}^{-1}$ and $1618 \mathrm{~cm}^{-1}$ can still be identified even for $5 \mu \mathrm{L}$ of $1 \mathrm{nM}$ MG (1.82 pg). The linear relationship between SERS intensity $I$ and MG concentration $C$ at $1-500 \mathrm{nM}$ was shown in Fig. 3D. The linear equation at $1618 \mathrm{~cm}^{-1}$ was $I_{1}=174.32 C+2107.90\left(R^{2}=0.9362\right)$. The RSD $\%$ at each concentration was found to be within 9.53-23.34\%. The LOD was found to be $0.4 \mathrm{nM}$ $(0.73 \mathrm{pg})$. The linear equation at $1175 \mathrm{~cm}^{-1}$ was
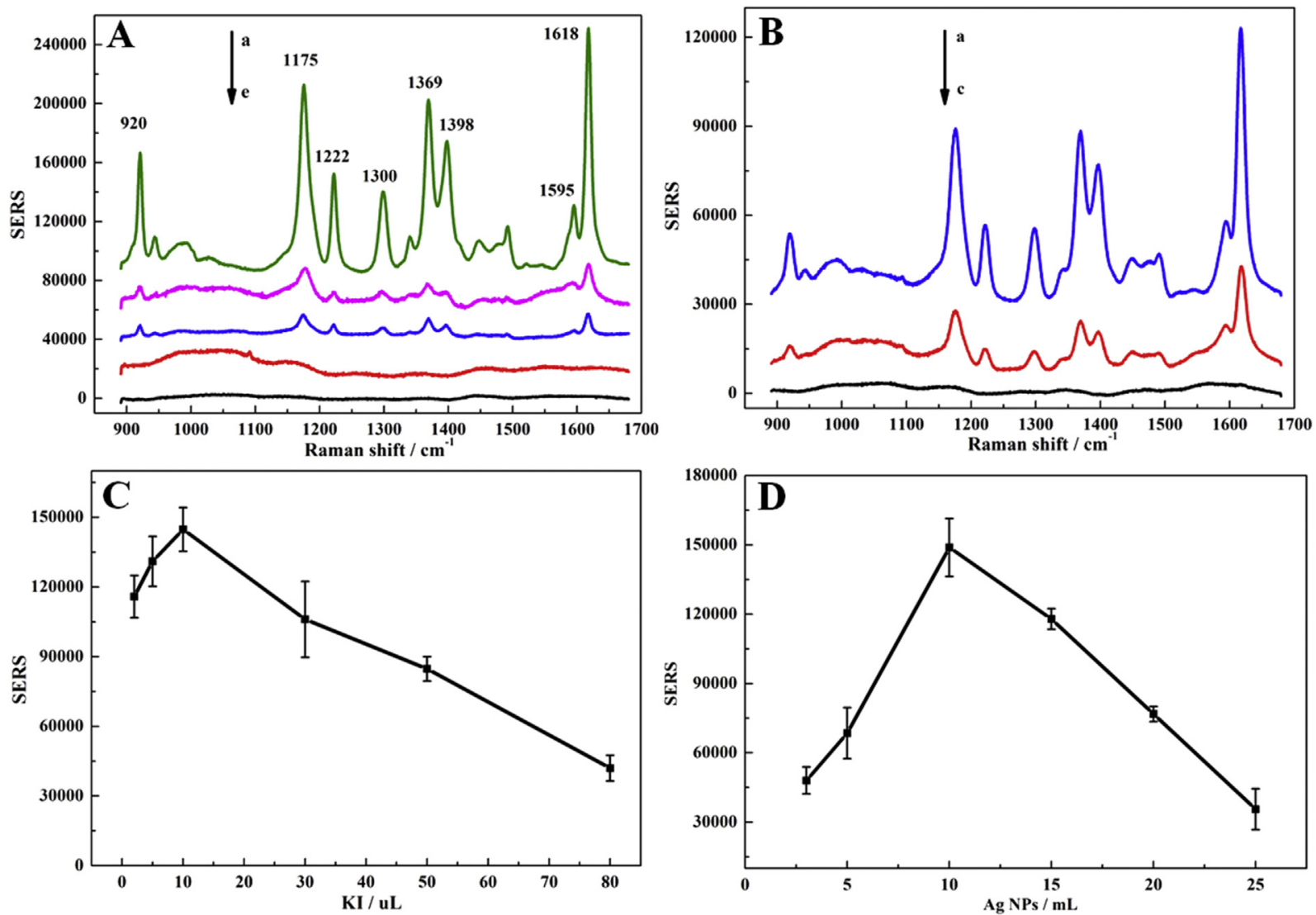

Fig. 2. A: The SERS spectra of MG with G-SERS sensor (a), MG with Ag NPs and gum mixture (b), MG with Ag NPs and KI mixture (c), MG with KI and gum mixture (d) and G-SERS sensor (e). B: The effect of halogen ions (10 $\mu \mathrm{L} 100 \mathrm{mM})$. Spectra (a) to (c) were KI, KBr and KCl, respectively. C: SERS performance of G-SERS sensor $(0.3 \mathrm{~g})$ modified with different $K I: 2,5,10,20,30,50$, and $80 \mu L$, respectively. D: SERS performance of G-SERS sensor modified with different amount of Ag NPs: 3, 5, 10, 15, 20, and $25 \mathrm{~mL}$, respectively. $5 \mu \mathrm{L}$ of $1 \mu \mathrm{M}$ MG was used as the Raman probe. Laser power: $12 \mathrm{~mW}$. Integration time: 5 s. Objective: $50 \times$. 
$I_{2}=126.19 \mathrm{C}+1689.78\left(R^{2}=0.9553\right)$. The RSD $\%$ at each concentration was found to be $9.03-24.50 \%$. To further prove its sensitivity, R6G and NBA were also examined, and the LODs were both $5 \mathrm{fmol}$ (Fig. S4), indicating the G-SERS sensor had good sensitivity.

\subsection{The sampling recovery efficiency (SRE) of the G-SERS sensor}

We evaluated the SRE of MG on the fish by the GSERS sensor (Fig. S2). The value was found to be $72.30 \%$, showing that the G-SERS sensor had good recovery efficiency [35]. The possible reasons for the relatively low collection efficiency were the rough surface of the fish and the permeation of MG into the fish.

\subsection{Detection of MG on the fish skin}

Owing to the good flexibility, stability, and recovery efficiency of gum sensors, it could be applied for rapid, direct, and on-site detection of MG on the live fish. Briefly, the prepared G-SERS sensor was used to stamp on the live fish (Fig. S1B) each day, and the SERS spectra were recorded. The results from one of the fish were shown in Fig. 4A. It was found that the blank fish had no SERS features of MG, and the signal of MG on the spiked fish in tap water decreased with time. The peak of residual MG on the spiked fish at $1618 \mathrm{~cm}^{-1}$ and $1175 \mathrm{~cm}^{-1}$ on the sixth day in tap water still could be detected. We suspected that the signal of MG gradually decreased because some MG on the spiked fish partly converted to leucomalachite green (LMG) [36] and/or partly dissolved in water. The remaining results from other fish were shown in Figs. S5-S11. Note that the SERS signal of MG residue on fish skin could not be found with the G-SERS sensor after the seventh day, while it was still present in fish tissue (Table S2). This could be justified by the two sampling methods. The G-SERS is intended to monitor the MG residue on fish skin, while the LC-MS examines the fish tissue. Since the proposed G-SERS method is intended to safeguard any potential MG adulteration happening after leaving the farm and before the dining table, which is usually less than 1 week, we believe the proposed method is reliable.
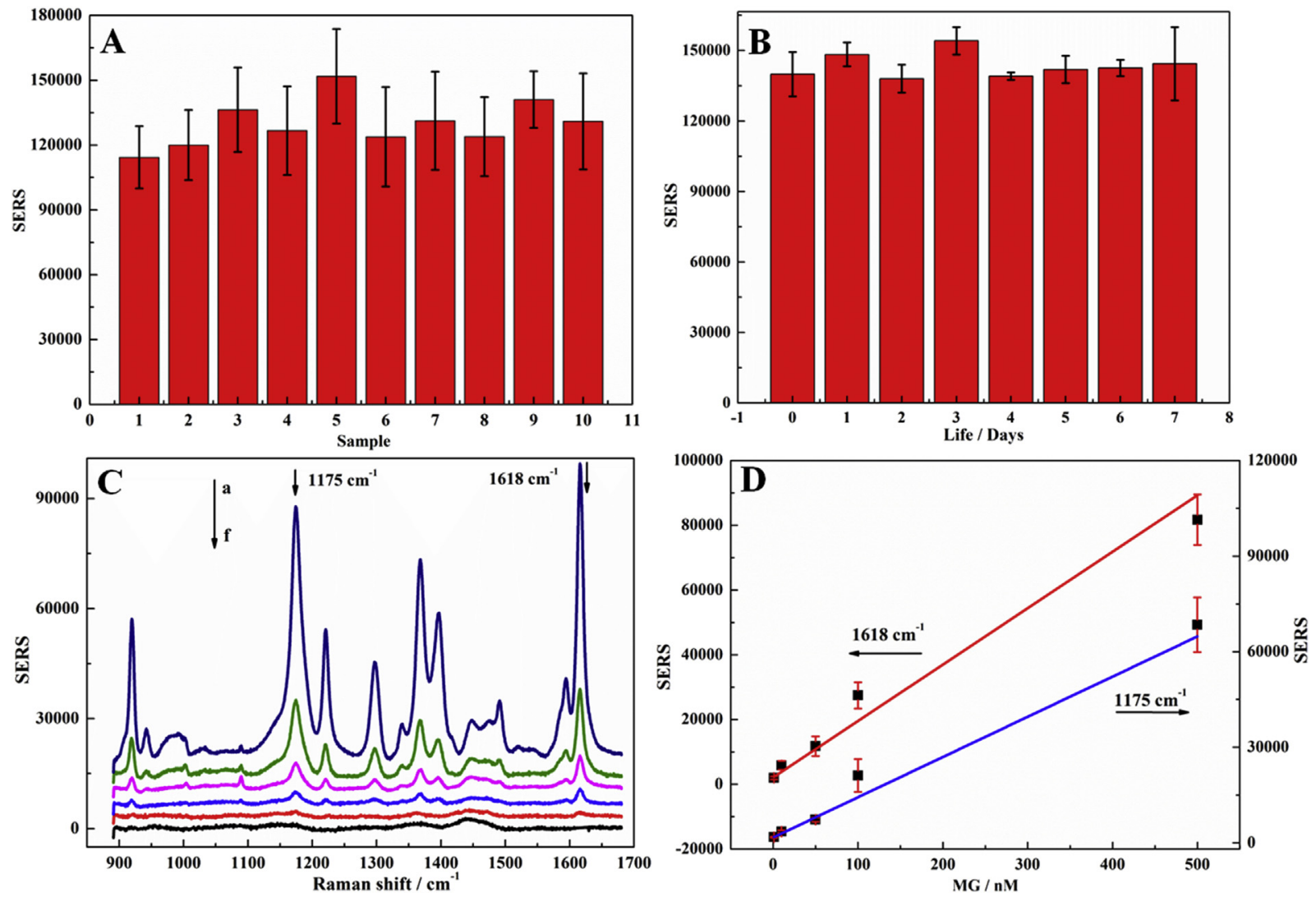

Fig. 3. A: Statistics of $10 \mathrm{G}-\mathrm{SERS}$ sensors obtained by using $5 \mu L$ of $1 \mu M$ MG as a Raman probe. B: Statistics of $5 \mu L$ of $1 \mu M$ MG aqueous solution on the G-SERS sensor corresponding to the signal variation every day in 8 days. 0 is that the sensor was prepared and used on the same day. C: The SERS spectra of different amount of MG: 500 (a), 100 (b), 50 (c), 10 (d), 1 (e), and 0 (f) $n M$, respectively. D: The plots of SERS intensity at characteristic peaks of 1175 and $1618 \mathrm{~cm}^{-1}$ with different concentrations of MG, respectively. 

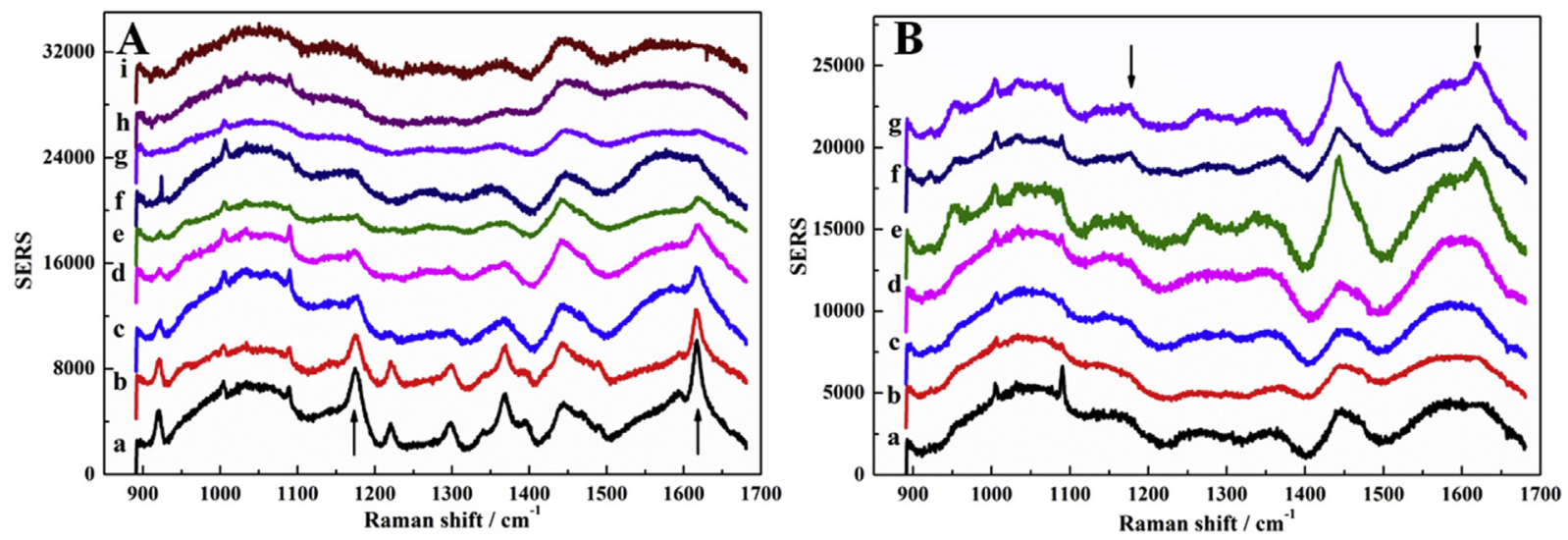

Fig. 4. A: The SERS spectra of MG on carp: crucian carp exposed to 0.5 ppm MG aqueous solution for 1 day (a), then raised in tap water for 1 (b), 2 $(c), 3(d), 4(e), 5(f), 6(g)$ days, the blank fish (h) and G-SERS sensor (i), respectively. B: The SERS spectra obtained on live fish from a supermarket and a street marketplace: spectrum (a) was G-SERS sensor, spectra (b) to (c) were two crucian carp from the supermarket, and spectra (d) to $(g)$ were four crucian carp from the same street marketplace (four stalls), respectively.

Table 1. Quantitative and confirmatory analysis of MG in fish by LCMS.

\begin{tabular}{llll}
\hline Sample & \multicolumn{2}{l}{ LC-MS (LOD =0.5 ug/kg) } & SERS \\
\cline { 2 - 3 } & mean (ug/kg) & RSD (\%) & \\
\hline Street marketplace (stall 1) & 50.30 & 1.14 & Positive \\
Street marketplace (stall 2) & 37.35 & 1.74 & Positive \\
Street marketplace (stall 3) & 28.28 & 2.75 & Positive \\
Street marketplace (stall 4) & Not found & $/$ & Negative \\
Supermarket 1 & Not found & $/$ & Negative \\
Supermarket 2 & Not found & $/$ & Negative \\
\hline
\end{tabular}

We finally applied the proposed method to detect MG on the live fish from the local supermarket and street marketplace (four stalls), the results were shown in Fig. 4B. It was found that the peak of MG on the fishes from the street marketplace at 1175 and $1618 \mathrm{~cm}^{-1}$ could be identified, while that from the supermarket could not. As shown in Table 1 and Fig. S12, LC-MS was used to quantify the amount of MG in the samples. The results coincided with the results of SERS analysis. In other words, the SERS method could be used to monitor MG contamination risks on live fish.

\section{Conclusion}

In conclusion, a novel dual functional gum based flexible SERS sensor (G-SERS) was proposed for monitoring MG contamination risks on live fish during shipping and in the marketplaces. The combination of iodide ion and chewing gum helps the signal enhancement on Ag NPs, making it possible for the detection of MG at $0.73 \mathrm{pg}$. The flexibility and viscoelasticity of the chewing gum make it possible for the direct sampling of a minute amount of MG on fish skin. To illustrate the effectiveness in food safety screening of the proposed G-SERS sensor, crucian carp was raised in $0.5 \mathrm{ppm}$ MG solution for 1 day, and then in fresh water for another 6 days to mimic the live fish shipping and sale cycle, and monitored daily for the presence of MG. SERS signal of the residual MG on fish could be still found even after 6 days in fresh water. Crucian carp from the local supermarket and street marketplace was also screened and crossvalidated with LC-MS. Positive results were found on some fishes from the local street marketplace, suggesting MG risks still exist in smaller marketplaces.

\section{Conflict of interest}

The authors of the manuscript declare that there is no conflict of interest regarding the publication of this study.

\section{Acknowledgments}

The authors acknowledge the financial support from the National Natural Science Foundation of China (no. 21677117 and no. 21777131), Science and Technology Project of Sichuan Province (no. 2017GZ0388) and Doctoral Innovation Fund Program of Southwest Jiaotong University (No. DCX201801).

\section{Appendix A. Supplementary data}

Supplementary data to this article can be found online at https://doi.org/10.38212/2224-6614.1057.

\section{References}

[1] Zhou B, Shen J, Li P, Ge M, Lin D, Li YY, et al. Gold nanoparticle-decorated silver needle for surface-enhanced Raman spectroscopy screening of residual malachite green 
in aquaculture products. ACS Appl Nano Mater 2019;2: 2752-7.

[2] Pepper IL, Brooks JP, Gerba CP. Antibiotic resistant bacteria in municipal wastes: is there reason for concern? Environ Sci Technol 2018;52:3949-59.

[3] Liu S, Dong G, Zhao H, Chen M, Quan W, Qu B. Occurrence and risk assessment of fluoroquinolones and tetracyclines in cultured fish from a coastal region of northern China. Environ Sci Pollut Res 2018;25:8035-43.

[4] Done HY, Halden RU. Reconnaissance of 47 antibiotics and associated microbial risks in seafood sold in the United States. J Hazard Mater 2015;282:10-7.

[5] Levin-Reisman I, Ronin I, Gefen O, Braniss I, Shoresh N, Balaban NQ. Antibiotic tolerance facilitates the evolution of resistance. Science 2017;355:826-30.

[6] Huang W, Yang H, Zhang S. Acetylacetone extends the working life of laccase in enzymatic transformation of malachite green by interfering with a key intermediate. J Hazard Mater 2019;366:520-8.

[7] Sudova EMJ, Svobodova Z, Vesely T. Negative effects of malachite green and possibilities of its replacement in the treatment of fish eggs and fish: a review. Vet Med-CZECH 2007; 12:527-39.

[8] Ouyang L, Yao L, Zhou T, Zhu L. Accurate SERS detection of malachite green in aquatic products on basis of graphene wrapped flexible sensor. Anal Chim Acta 2018; 1027:83-91.

[9] Lim LCD P, Sorgeloos P. In: Preparation for transport: fish packaging Technology, 2. Maarseen, the Netherlands: Ornamental Fish International Educational Publication; 2007. p. 25-45.

[10] Mitrowska K, Posyniak A, Zmudzki J. Determination of malachite green and leucomalachite green in carp muscle by liquid chromatography with visible and fluorescence detection. J Chromatogr A 2005;1089:187-92.

[11] Garcia-Rico E, Alvarez-Puebla RA, Guerrini L. Direct surface-enhanced Raman scattering (SERS) spectroscopy of nucleic acids: from fundamental studies to real-life applications. Chem Soc Rev 2018;47:4909-23.

[12] Chen J, Huang M, Kong L, Lin M. Jellylike flexible nanocellulose SERS substrate for rapid in-situ non-invasive pesticide detection in fruits/vegetables. Carbohydr Polym 2019;205:596-600.

[13] Zhong L-B, Yin J, Zheng Y-M, Liu Q, Cheng X-X, Luo F-H. Self-assembly of Au nanoparticles on PMMA template as flexible, transparent, and highly active SERS substrates. Anal Chem 2014;86:6262-7.

[14] Zhan H, Cheng F, Chen Y, Wong KW, Mei J, Hui D, et al. Transfer printing for preparing nanostructured PDMS film as flexible SERS active substrate. Compos B Eng 2016;84:222-7.

[15] Sun H, Liu H, Wu Y. A flexible and highly sensitive SurfaceEnhanced Raman Scattering film in-situ detection of malachite green on fish skin. Mater Lett 2017;207:125-8.

[16] Kumar P, Khosla R, Soni M, Deva D, Sharma SK. A highly sensitive, flexible SERS sensor for malachite green detection based on Ag decorated microstructured PDMS substrate fabricated from Taro leaf as template. Sensor Actuator B Chem 2017;246:477-86.

[17] Yang N, You T-T, Gao Y-K, Zhang C-M, Yin P-G. Fabrication of a flexible gold nanorod polymer metafilm via a phase transfer method as a SERS substrate for detecting food contaminants. J Agric Food Chem 2018;66:6889-96.

[18] Zhou Y, Li X, Pan Z, Ye B, Xu M. Determination of malachite green in fish by a modified MOF-based electrochemical sensor. Food Anal Method 2019;12:1246-54.

[19] Su L, Xiong Y, Chen Z, Duan Z, Luo Y, Zhu D, et al. $\mathrm{MoO}_{3}$ nanosheet-assisted photochemical reduction synthesis of $\mathrm{Au}$ nanoparticles for surface-enhanced Raman scattering substrates. Sensor Actuator B Chem 2019;279:320-6.
[20] Zhang H, Zhang W, Gao X, Man P, Sun Y, Liu C, et al. Formation of the AuNPs/GO@MoS $/$ AuNPs nanostructures for the SERS application. Sensor Actuator B Chem 2019;282: 809-17.

[21] Yao J, Quan Y, Gao M, Gao R, Chen L, Liu Y, et al. AgNPs decorated $\mathrm{Mg}$-doped $\mathrm{ZnO}$ heterostructure with dramatic SERS activity for trace detection of food contaminants. J Mater Chem C 2019;7:8199-208.

[22] Zhang M, Chen Z, Wang Z, Zheng Z, Wang D. Graphene oxide coated popcorn-like Ag nanoparticles for reliable sensitive surface-enhanced Raman scattering detection of drug residues. J Mater Res 2019;34:2935-43.

[23] Zhang $M$, Yang J, Wang $Y$, Sun $H$, Zhou $H$, Liu X, et al. Plasmon-coupled 3D porous hotspot architecture for supersensitive quantitative SERS sensing of toxic substances on real sample surfaces. Phys Chem Chem Phys 2019;21: 19288-97.

[24] Zhang D, You H, Yuan L, Hao R, Li T, Fang J. Hydrophobic slippery surface-based surface-enhanced Raman spectroscopy platform for ultrasensitive detection in food safety applications. Anal Chem 2019;91:4687-95.

[25] Islam AM, Phillips GO, Sljivo A, Snowden MJ, Williams PA. A review of recent developments on the regulatory, structural and functional aspects of gum Arabic. Food Hydrocolloids 1997;11:493-505.

[26] Darabi MA, Khosrozadeh A, Wang Q, Xing M. Gum sensor: a stretchable, wearable, and foldable sensor based on carbon nanotube/chewing gum membrane. ACS Appl Mater Interfaces 2015;7:26195-205.

[27] Gong Z, Wang C, Wang C, Tang C, Cheng F, Du H, et al. A silver nanoparticle embedded hydrogel as a substrate for surface contamination analysis by surface-enhanced Raman scattering. Analyst 2014;139:5283-9.

[28] Andersen WC, Turnipseed SB, Roybal JE. Quantitative and confirmatory analyses of malachite green and leucomalachite green residues in fish and shrimp. J Agric Food Chem 2006;54:4517-23.

[29] Roosta M, Ghaedi M, Shokri N, Daneshfar A, Sahraei R, Asghari A. Optimization of the combined ultrasonic assisted/ adsorption method for the removal of malachite green by gold nanoparticles loaded on activated carbon: experimental design. Spectrochim Acta 2014;118:55-65.

[30] Xu LJ, Zong C, Zheng XS, Hu P, Feng JM, Ren B. Label-free detection of native proteins by surface-enhanced Raman spectroscopy using iodide-modified nanoparticles. Anal Chem 2014;86:2238-45.

[31] Lai K, Zhang Y, Du R, Zhai F, Rasco BA, Huang Y. Determination of chloramphenicol and crystal violet with surface enhanced Raman spectroscopy. Sens \& Instrumen Food Qual 2011;5:19-24.

[32] Zhang Y, Yu W, Pei L, Lai K, Rasco BA, Huang Y. Rapid analysis of malachite green and leucomalachite green in fish muscles with surface-enhanced resonance Raman scattering. Food Chem 2015;169:80-4.

[33] An J, Tang B, Zheng X, Zhou J, Dong F, Xu S, et al. Sculpturing effect of chloride ions in shape transformation from triangular to discal silver nanoplates. J Phys Chem C 2008; 112:15176-82.

[34] Fan M, Andrade GFS, Brolo AG. A review on recent advances in the applications of surface-enhanced Raman scattering in analytical chemistry. Anal Chim Acta 2020;1097: $1-29$.

[35] Sun J, Gong L, Lu Y, Wang D, Gong Z, Fan M. Dual functional PDMS sponge SERS substrate for the on-site detection of pesticides both on fruit surfaces and in juice. Analyst 2018; 143:2689-95.

[36] Ran H, Lin Z-Z, Yao O-H, Hong C-Y, Huang Z-Y. Ratiometric fluorescence probe of MIPs@CdTe QDs for trace malachite green detection in fish. Anal Bioanal Chem 2019; 411:537-44. 